\title{
CHARACTERIZATION OF HIGH TEMPORAL RESOLUTION PRR ACQUISITION BY FAST COMTEC CARD: DEADTIME, PRR DESATURATION, TEMPERATURE CALIBRATION AND RETRIEVAL.
}

\author{
Giovanni Martucci $^{1}{ }^{*}$, Valentin Simeonov ${ }^{2}$, Ludovic Renaud ${ }^{1}$, and Alexander Haefele ${ }^{1}$ \\ ${ }^{I}$ Federal Office of Meteorology and Climatology, MeteoSwiss, Payerne, Switzerland, \\ *Giovanni.martucci@meteoswiss.ch \\ ${ }^{2}$ Ecole Polytechnique Federale de Lausanne - EPFL, CH-1015 Lausanne, Switzerland
}

\begin{abstract}
RAman Lidar for Meteorological Observations (RALMO) is operated at MeteoSwiss and provides continuous measurements of water vapor and temperature since 2010. While the water vapor has been acquired by a Licel acquisition system since 2008, the temperature channels have been migrated to a Fastcom P7888 acquisition system, since August 2015. We present a characterization of this new acquisition system, namely its dead-time, desaturation, temporal stability of the Pure Rotational Raman signals and the retrieval of the PRR-temperature.
\end{abstract}

\section{INTRODUCTION}

The real time and continuous monitoring of the temperature in the troposphere and lower stratosphere has both a meteorological and climatological importance. Accurate measurements of the atmospheric temperature are needed for numerical weather prediction and on the long term to understand the changes and trends of temperature for a climatological applications. The temperature can also be used, in combination with the water vapor, to calculate the relative humidity, a variable of prime importance to understand the dynamics of cloud formation and the hygroscopic growth of aerosols in the boundary layer.

In collaboration with the École Polytechnique Fédérale de Lausanne (EPFL), the Federal Office of Meteorology and Climatology MeteoSwiss operates a Raman LIDAR to measure water vapor and temperature in the troposphere and lower stratosphere. The RAman Lidar for Meteorological Observations (RALMO) is operated at the Aerological station at Payerne $\left(46^{\circ} 480 \mathrm{~N}, 6^{\circ} 560 \mathrm{E}, 491 \mathrm{ma} . \mathrm{s} .1.\right)$ by MeteoSwiss and provides continuous measurements of water vapor and temperature almost uninterruptedly since 2008 [1],[2]. While the water vapor has been acquired by the Licel acquisition system (licel.com/) since 2008, the Pure Rotational Raman (PRR) channels have been migrated to a new and faster acquisition system, the FAST ComTec P7888 (www.fastcomtec.com), since August 2015. This study presents a characterization of the acquisition system, namely its deadtime, the desaturation procedure of the high $(\mathrm{JH})$ and low (JL) rotational quantum number PRR channels based on the nonparalyzable condition and the temporal stability of the PRR signals. The retrieval procedure of the PRR-based temperature profile using different parameterizations accounting for higher-degree terms and its calibration based on radiosonde data are also discussed

\section{INSTRUMENT DESCRIPTION}

When RALMO has been designed, the main objectives of the project helped defining the system parameters. The main purpose was to measure water vapor and temperature profiles in the middle and high troposphere during day and night continuously. In order to achieve this target with profiles of high quality, the system has been created based on to the following specifications: a narrow receiver's field of view, a narrowband grating polychromator that isolates the rovibrational Q-branches allowing to minimize the contamination by solar background and to maximize the signal-to-noise ratio, a near realtime data availability, long-term data consistency and system stability, autonomous operation, minimal maintenance and eye safety to ensure the possibility to measure without interfering with the surroundings. Such a system ensures high accuracy and precision of the measured parameters, the traceability of the measurement and high data availability since 2008 . Table 1 summarizes the system parameters as it is 
described more in detail by Dinoev et al., in 2013 [1]. RALMO laser's source emits at $1064 \mathrm{~nm}$ its fundamental harmonic, but uses the third harmonic at $355 \mathrm{~nm}$, the tripled harmonic is then optically directed to the LIDAR transceiver optics. A beam expander reduces the beam divergence at the instrument's output and the expanded beam is transmitted into the atmosphere. The light backscattered by the atmosphere is collected by the LIDAR's telescope (4 mirrors of $30 \mathrm{~cm}$ diameter) and transmitted by optical fibers separately to the $\mathrm{H}_{2} \mathrm{O}$ and Aerosol/Temperature polychromators for the wavelength separation. The photo multipliers detect the optical signals and convert them into electrical signals, which are acquired and stored for the data analysis.

Table 1 RALMO system parameters

\begin{tabular}{c|c}
\hline Transmitter & Receiver \\
\hline Nd:YAG laser - third & Four fiber-coupled \\
harmonic: & parabolic mirrors: \\
Wavelength: $354.7 \mathrm{~nm}$ & Focal length: $1 \mathrm{~m}$ \\
Spectral line width: 0.7 & Diameter: $0.3 \mathrm{~m}$ \\
cm-1 & Axial displacement: \\
Rep. rate: $30 \mathrm{~Hz}$ & $235 \mathrm{~mm}$ \\
Pulse energy: $300 \mathrm{~mJ}$ & FOV: $3 \times 0.20$ and $1 \times 0.22$ \\
Pulse duration: $8 \mathrm{~ns}$ & mrad \\
Beam expander - Galilean & \\
type: & Two-stage, grating \\
Expansion ratio $15 \times$ & Polychromator \\
Transmitted beam: & Photodetectors \\
Divergence: & (Hamamatsu) \\
calculated $0.06 \mathrm{mrad}$ & R12421 \\
measured $0.09 \pm 0.02 \mathrm{mrad}$ & \\
Diameter: $140 \mathrm{~mm}$ &
\end{tabular}

\section{$3 \quad$ FAST ComTec P7888}

The Model P7888 Series is one of the fastest commercially available multiple-event time digitizer with up-to four inputs. P7888 has been installed to acquire the PRR RALMO channels in August 2015 to reduce significantly the acquisition system's deadtime and consequently to minimize the saturation effects in the photoncounting channel. Full specifications of the FAST ComTec P7888 acquisition card can be found at www.fastcomtec.com. Compared to the Licel acquisition system, the P7888 acquires the PRR channels with higher range resolution and with about twice shorter deadtime, $\tau$. The new acquisition system uses solely the photon counting method with two transmissivity channels (high and low) for $\mathrm{JH}$ and JL. The high-transmission channel corresponds to the $90 \%$ of the signal intensity, while the low-transmission corresponds to the remaining $10 \%$. The low-transmission channels $\left(\mathrm{JH}^{10 \%}, \mathrm{JL}^{10 \%}\right)$ are theoretically never saturated and can act as reference unsaturated channel when they are linearly compared with the high-transmission channels $\left(\mathrm{JH}^{90 \%}, \mathrm{JL}^{90 \%}\right)$. In order to calculate the count rate threshold at which saturation starts to occur and the corresponding deadtime, we have studied the departure from linearity of the high-transmission channel. For over a year of data acquired by the P7888 card we have characterized the values of $\tau$, when the saturation was directly observable.
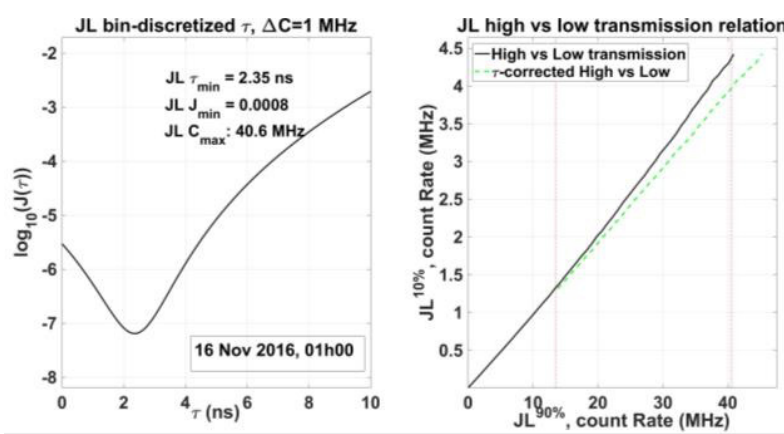

Figure 1 Deadtime calculation and high-transmission channel deadtime correction.

By applying the non-paralyzable assumption [3], the two signals $\mathrm{JH}^{90 \%}$ and $\mathrm{JL}^{90 \%}$ show saturation values as soon as the signal exceeds the threshold counts of about $35 \mathrm{MHz}(\sim 10 \mathrm{MHz}$ for Licel) with values of $\tau$ of $2 \mathrm{~ns}$ and $1.35 \mathrm{~ns}$, respectively. The method adopted to calculate $\tau$ and to desaturate the PRR channels is based on the non-linear relation between an unsaturated and a saturated channel and has been described by previous authors, e.g. [3], [4]. Figure 1 shows an example of calculation of the deadtime $\tau$ for the $\mathrm{JL}^{90 \%}$ signal obtained by minimization of the leastsquares matrix $\boldsymbol{J}$ (left panel) and the desaturation by use of the non-paralyzable assumption (right panel). The matrix $\boldsymbol{J}$ depends on the fit-error normalized differences between the deadtimecorrected count rates and the virtual count rates.

\section{PRR-BASED TEMPERATURE}

In addition to the Rayleigh light backscattered by the aerosols and molecules at the same frequency 
as the incident one, the $\mathrm{O}_{2}$ and $\mathrm{N}_{2}$ molecules returns a frequency-shifted Raman light to the LIDAR receiver. The returned signal is shifted in frequency due to the rotational and vibrational Raman effect. In this study only the pure rotational part of the spectrum around the Rayleigh frequency is analyzed (Fig. 2).

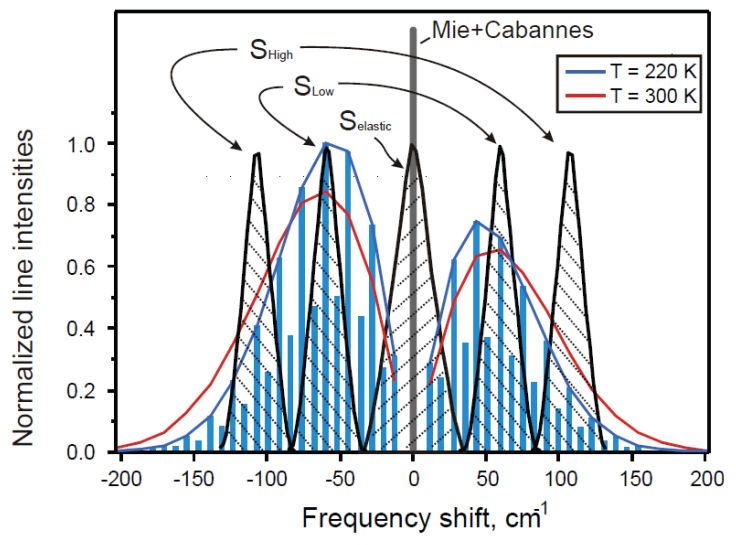

Figure 2 Temperature dependence of the purerotational Raman spectrum of $\mathrm{N}_{2}[6]$

The high-frequency and low-frequency shifted signals in the Stokes and anti-stokes Q-branches depend on the temperature of the probed atmospheric volume. The temperature $T$ at distance $z$ is derived from the ratio of the intensity of the PRR signals JL and $\mathrm{JH}$ at $\mathrm{z}$, using the following approximate relationship [5], where the constants $A$ and $B$ are determined during the LIDAR calibration.

$$
T(z)=\frac{A}{\log \left(\frac{J L(z)}{J H(z)}\right)+B}
$$

\subsection{Nonlinear parameterizations}

The temperature retrieval accuracy and number of coefficients as well as the degree of the terms depending on the PRR ratio depends on the degree of the expanded Taylor's series of the $\log (\mathrm{JL} / \mathrm{JH})$ ratio. The generally-used parameterization has a linear dependence on the logarithmic term $\mathrm{JL} / \mathrm{JH}$, which simplifies the retrieval and the calibration of the temperature, but that ignores the broadening of individual atmospheric $\mathrm{N}_{2}$ and $\mathrm{O}_{2}$ PRR lines, contributing to the overall uncertainty of the retrieval. Moreover, the theoretical expression of eq.(1) refers strictly to a single line of the PRR spectrum, and the fact of using it for multiple spectral lines introduces an additional uncertainty. The overall uncertainty related to the coupled system retrieval + instrument, where the instrument contributes with the measurement noise, is on the order of $\pm 1 \mathrm{~K}$ [7], [8]. The pressure broadening of $\mathrm{N}_{2}$ and $\mathrm{O}_{2}$ PRR lines dominates over other types of broadening in the troposphere, and it should not be neglected when higher-degree terms are taken into account for the retrieval of the temperature. When expanding the Taylor's series at higher degrees other two parameterizations can be used as suggested by previous authors, i.e., [8],[9].

$$
\begin{gathered}
T=\frac{A}{\log \left(\frac{J L}{J H}\right)^{2}+B \log \left(\frac{J L}{J H}\right)+C} \\
T=\frac{\log \left(\frac{J L}{J H}\right)}{A \log \left(\frac{J L}{J H}\right)^{2}+B \log \left(\frac{J L}{J H}\right)+C}
\end{gathered}
$$

Especially Eq. (3) has proved to provide better calibrations of the temperature profiles [8] and a reduced related error. We have tested over one year of RALMO PRR data, retrieving the temperature using equations 1-3: the results proved a good agreement with the findings of [9], showing better calibrations using equations 2 and 3 , but an unsatisfactory reproducibility of the calibration coefficients over the days and weeks following a calibration.

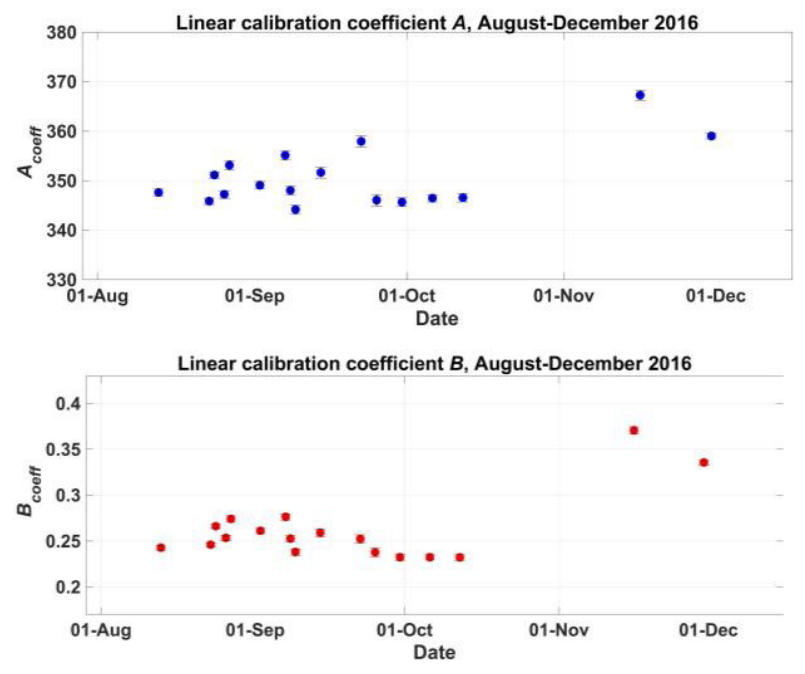

Figure 3 A timeseries of and B calibration coefficients

Finally eq. (1) leads to a slightly larger related error in the temperature retrieval $(\sim 1 \mathrm{~K})$ compared 
to eqs. 2-3, but the retrieved calibration coefficients can be used robustly over several weeks, allowing for a more stable and homogeneous temperature time series. We have then decided to use only eq. (1) to retrieve $T$.

In Figure 3 we show the variability of the two calibration coefficients $A$ and B based on eq. (1) over 3 months. The standard deviation of $A$ and $B$ with relation to their mean values is $1.75 \%$ and $14.24 \%$, respectively. The $T$ profiles retrieved from RALMO profiles using eq.(1) and compared with the radiosounding (RS) after calibration have provided, for the period September-December 2016 (19 profiles), a statistical bias (RALMO-RS) and standard deviation (stdv) that are shown in Figure 4.

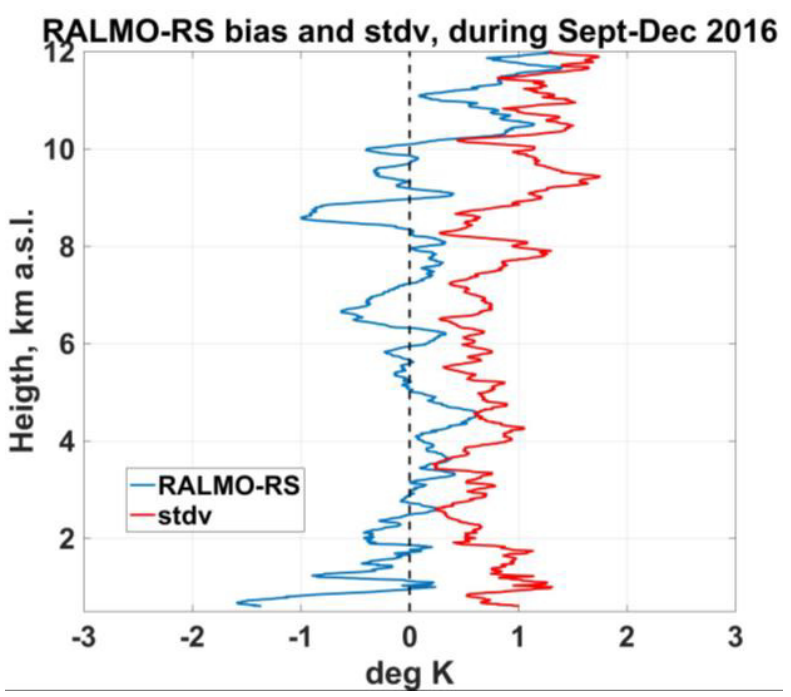

Figure 4 Bias and standard deviation (stdv) values of RALMO-RS during September-December 2016.

The mean bias does not exceed the $[-1,+1] \mathrm{K}$ interval, showing a cold bias of $-1 \mathrm{~K}$ in the first few hundred meters. The RMSE is about $1 \mathrm{~K}$ up to $8 \mathrm{~km}$ and reaches $2 \mathrm{~K}$ between 8 and $12 \mathrm{~km}$.

\section{CONCLUSIONS}

The high temporal resolution PRR acquisition card by FAST ComTec P7888, has been characterized for its deadtime for both the $\mathrm{JH}$ and JL PRR channels. The deadtime, compared to the Licel deadtime is shorter, which implies a reduced saturation of the PRR signals. Different parameterizations have been tested to calibrate the temperature profile retrieved from the PRR signals. The linear parameterization in eq. (1) provides the best compromise between accuracy and reproducibility of the calibration coefficients. Finally a 3-month statistic of stability of the calibration coefficients and the bias and standard deviation of the temperature values based on the radiosounding temperature profiles has been performed. The statistics show that the bias is restricted within the interval $[-1,+1] \mathrm{K}$.

\section{References}

[1] Dinoev, T., Simeonov, V., Arshinov, Y., Bobrovnikov, S., Ristori, P., Calpini, B., Parlange, M., and van den Bergh, H.: Raman Lidar for Meteorological Observations, RALMO - Part 1: Instrument description, Atmos. Meas. Tech., 6, 1329-1346, doi:10.5194/amt-6-1329-2013, 2013

[2] Brocard, E., Philipona, R., Haefele, A., Romanens, G., Ruffieux, D., Simeonov, V., and Calpini, B.: Raman Lidar for Meteorological Observations, RALMO - Part 2: Validation of water vapor measurements, Atmos. Meas. Tech.,6, 1347-1358, doi:10.5194/amt-6-13472013, 2013.

[3] Whiteman, D. N.: Raman lidar system for the measurement of water vapor and aerosols in the Earth's atmosphere, Appl. Opt., 31, 3068-3082, 1992.

[4] Newsom R K, Turner D D, Mielke B, et al, 2009: Simultaneous analog and photon counting detection for Raman lidar. Applied optics, 2009, 48(20), 3903-3914.

[5] Arshinov, Y. F., Bobrovnikov, S. M., Zuev, V. E., and Mitev, V. M.: Atmospheric temperature measurements using a pure rotational Raman lidar, Appl. Optics, 22, 2984-2990, 1983.

[6] Dinoev, T. S., V. B. Simeonov, B. Calpini, and M. Parlange, "Monitoring of Eyjafjallajökull ash layer evolution over payerne Switzerland with a Raman lidar," in Proceedings of the TECO, Helsinki, Finland, 2010.

[7] Behrendt, A., T. Nakamura, M. Onishi, et al.: Appl. Opt., 41, 7657, 2002.

[8] Zuev, V. V., Gerasimov, V. V., Pravdin, V. L., Pavlinskiy, A. V., and Nakhtigalova, D. P.: Atmos. Meas. Tech., 10, 315-332, doi:10.5194/amt-10-315-2017, 2017.

[9] Behrendt A., and J. Reichardt, Appl. Opt. 39, 1372-1378, 2000. 